\title{
DYNAMICAL INVARIANTS FOR CPG CONTROL IN AUTONOMOUS ROBOTS
}

\author{
Fernando Herrero-Carrón, Francisco de Borja Rodríguez and Pablo Varona \\ Grupo de Neurociencia Computacional, Escuela Politécnica Superior, Universidad Autónoma de Madrid \\ Calle Francisco Tomás y Valiente 11, 28049, Madrid, Spain \\ \{fernando.herrero, f.rodriguez, pablo.varona\}@uam.es
}

Keywords: Bio-inspired robotics, Central pattern generators.

\begin{abstract}
Several studies have shown the usefulness of central pattern generator circuits to control autonomous rhythmic motion in robots. The traditional approach is building CPGs from nonlinear oscillators, adjusting a connectivity matrix and its weights to achieve the desired function. Compared to existing living CPGs, this approach seems still somewhat limited in resources. Living CPGs have a large number of available mechanisms to accomplish their task. The main function of a CPG is ensuring that some constraints regarding rhythmic activity are always kept, surmounting any disturbances from the external environment. We call this constraints the "dynamical invariant" of a CPG. Understanding the underlying biological mechanisms would take the design of robotic CPGs a step further. It would allow us to begin the design with a set of invariants to be preserved. The presence of these invariants will guarantee that, in response to unexpected conditions, an effective motor program will emerge that will perform the expected function, without the need of anticipating every possible scenario. In this paper we discuss how some bio-inspired elements contribute to building up these invariants.
\end{abstract}

\section{INTRODUCTION}

Biology has been a source of inspiration for robot constructors for some time now. In particular, many works have built Central Pattern Generator circuits to generate rhythmic motion in a robot (see (Ijspeert, 2008) for a review). Usually CPGs are regarded as a black box dynamical system capable of generating rhythmic patterns, in a way that is flexible enough to accommodate external modulation and/or perturbations, but robust enough to stay close to an effective pattern.

Living CPGs have developed to perform very specific tasks involving rhythmic activity: controlling swimming, chewing, breathing, heart beating, walking, etc (Grillner, 2006). But, not every individual of one same species presents the same physiological structures: due to differences in size, weight, or even history (illnesses, lesions, etc.) CPGs must be able to adapt to different environments (Bucher et al., 2005). Therefore, we can conclude that underlying CPG circuits there are mechanisms that allow for a great degree of flexibility while preserving its core function.

The core function of a CPG can be expressed as a set of dynamical invariants that must be preserved. Recent research (Reyes et al., 2008) has clearly shown that the pyloric CPG of the lobster presents substantial differences from animal to animal, yet robustly preserves the relationship between bursting period and phase lag between neurons. Even when one single CPG is forced by replacing one synapse with an artificially modified synapse, the CPG is shown to have some mechanism by which this relationship is preserved.

Traditional techniques to build CPG control in robots try to solve motor control tasks by mimicking known biological systems, in particular circuit topology and the presence of oscillators. This approach provides limited capability to deal with unforeseen scenarios. The concept of the dynamical invariant allows for a new design strategy in which the motor control can be achieved in a way that is not specified a priori, but emerges from the set of biologically inspired principles. Having a general set of invariant preserving mechanisms allows for more freedom in the process of design, providing more flexible and general solutions to existing problems in robot control. 


\section{INGREDIENTS FOR DYNAMICAL INVARIANT PRESERVING CPGS}

In this section we discuss scientific results on the mechanisms known to contribute to the essential flexibility and robustness of CPGs, which may be directly related to the implementation of dynamical invariants.

\subsection{Topologies}

CPGs are known for their flexibility: they generate a robust rhythmic activity with a recognizable pattern, but they can be modulated by external input. The final shape of the rhythm produced by one CPG will depend on many factors: intrinsic properties of individual neurons and synapses, strength and sign of the couplings and network topology, all contribute to the function of the circuit. It is known (Huerta et al., 2001; Stiesberg et al., 2007) that non-open topologies maximize the quality of the rhythm produced in terms of flexibility and regularity. A non-open topology is that in which every neuron receives at least one connection from another CPG member, in contrast to an "open" topology, where at least one neuron does not receive synapses from any other CPG member

In the design of an artificial CPG for robot control, it is necessary to take this into account. All neurons within the circuit must receive feedback from the rest of the circuit, so that knowledge about how the CPG is performing is distributed to all units. Of course, not all neurons will process this information in the same way.

Different works, both theoretical and experimental, have studied the role of synapses in the synchronization of neural oscillators. However, this study cannot be decoupled from the properties of the units being connected. Depending on the intrinsic characteristics of neurons, synaptic activity will have different effects. In fact, different CPGs may adopt different combinations of neural and synaptic properties to achieve the same goal (Prinz et al., 2004). The result is that the CPG designer has a wider variety of mechanisms to choose from. For instance, it is widely accepted that in-phase synchronization can be achieved through excitatory interaction, but it can also be achieved by inhibitory interaction with the appropriate conditions (Wang and Rinzel, 1992). And conversely, anti-phase synchronization is usually considered to happen under inhibition (Wang and Rinzel, 1992; Rowat and Selverston, 1997) but it can be found to happen under excitatory connections as well (Kopell and Somers, 1995).
Usually phase relationship between modules of a CPG is an important dynamical invariant that must be preserved. There exist some important theoretical studies on the mechanisms for phase locking between oscillators. A general study based on a phase model can be found in (Kopell and Ermentrout, 2000). However, it poses strong restrictions upon the coupling between oscillators. More realistic approaches that take into account synaptic dynamics for predicting the type of synchronization among bursting neurons can be found in (Oprisan et al., 2004) and (Elson et al., 2002).

When building complex artificial CPGs, it is difficult to predict how the coupling of neurons within a given topology will affect synchronization. Being able to predict stable phase-locking regimes as in the above mentioned works could help us design complex CPGs, with rich dynamics and guarantee that the resulting phase and frequency relationships will be the desired ones.

\subsection{Starting, Stopping and Maintenance of Rhythm}

Few studies in robotics are concerned with how CPGs are started and stopped. There exist various mechanisms underlying the starting, stopping and sustaining of rhythm in living CPGs.

In cases like breathing or heart beating, the group of neurons responsible for the maintenance of rhythm cannot afford to stop. There are other cases, however, in which a set of neurons needs to be activated and, after some time of activity, deactivated.

In the first case, special neurons called "pacemaker" neurons are responsible for setting the pace at which the rest of the group will oscillate (Selverston et al., 2009). Thanks to biophysical sub cellular mechanisms, they can produce oscillations in isolation, without external input. Through synaptic connections, the activity of pacemakers is "distributed" to other members of the groups.

Pacemaker neurons can usually adapt their burst frequency over a large range. By means of external input and feedback from other neurons in the network, the pacemaker group will be able to decide if the circuit is working properly and, if not, set the appropriate frequency. Some CPGs show redundancy mechanisms in order to maintain the correct rhythm in case of failure. Several neuron models are capable of endogenous oscillation as seen in living CPG neurons: many variations of the bio physically detailed model by Hodgkin and Huxley (Hodgkin and Huxley, 1952); one classical model for bursting activity (Hindmarsh and Rose, 1984) and other recent models with less 
computational requirements (Rulkov, 2002; Aguirre et al., 2005).

In the second case, initiation and termination are usually caused by external forces. A silent group of neurons may be "recruited" by an external excitatory force. Such is the case in (Arshavsky et al., 1998), where neuron group IN 12 is recruited by neuron group IN 8 and inhibited by neuron group IN 7 . This a very good example in which neurons that do not generate a rhythm autonomously contribute to the proper functioning of the CPG.

Interestingly, this CPG presents another important feature. Depending on the intensity of the swimming, an early group of neurons is recruited for weak swimming and if more powerful strokes are needed, a neuron group with higher activation threshold will also be recruited. This is the delayed group of neurons.

Another mechanism found in some neurons is known as "post-inhibitory rebound". This feature is widely observed, in particular again in (Arshavsky et al., 1998). A neuron with this feature will remain silent while inhibited. If inhibition is suddenly released, the neuron will respond with a spike or burst of activity.

It is believed that the selection of motor programs may be subject to an inhibitory mechanism (Grillner et al., 2005). Each possible motor program is kept under inhibition, until upper control centers decide to activate it. At this point, inhibition is released. By the mechanism of post-inhibitory rebound, a motor program could immediately start upon lifting of inhibition. Note that this is not equivalent to activation by excitation. The difference is that in a normal state, the circuit responsible of the motor program will not spontaneously activate because of noise, since inhibition will keep it forcibly silent.

The mechanism for termination of activity will depend on the goal of the CPG. If neurons in the CPG are not capable of endogenous oscillation, they may just go silent by themselves after activity has been elicited by one of the mechanisms mentioned earlier. If the activity needs to be sustained, then mutual excitatory connections within a group of neurons will keep them firing. This is known as a "pool" of neurons.

So the repertoire for how activity is elicited, maintained and stopped is really ample. However, each mechanism has different subtleties, depending on the underlying cellular characteristics, on whether inhibition or excitation should be preferred, etc.

\subsection{Motor Command Coding}

Individual CPG neurons display a mainly bursting activity. Motor commands are encoded in some aspect of the neuron's activity, for instance in the frequency of the spikes, or on the precise timing between them. How information is extracted from this activity is not trivial (Brezina et al., 2000), but simple mechanisms can be used in robots.

The fact that rhythmic motor commands are encoded using bursting neurons has an advantage over the classical view of CPGs in robotics: the mechanisms that encode motor commands and those used for synchronization are decoupled. In the traditional view, one non-linear oscillator represents a whole CPG. Then, one variable of the oscillator is used as output to the controlled joint and to other oscillators that need to be synchronized. Bursting neurons have the ability to flexibly adapt the timing between bursts and still produce robustly reproducible bursting patterns. With this decoupling, the system gains simultaneously in robustness and flexibility. In addition, some bursting neurons have the ability to encode information relative to their identity and their context and send messages to neighbouring neurons, as we discuss in the following section.

\subsection{Neural Signatures}

Recent experiments have shown that CPG individual cells have neural signatures that consist of neuron specific spike timings in their bursting activity (Szucs et al., 2003). Model simulations indicate that neural signatures that identify each cell can play a functional role in the activity of CPG circuits (Latorre et al., 2006). These signatures coexist with the information encoded in the slow wave rhythm of the CPG which results in a neural signal with multiple simultaneous codes. Readers of this signal (muscles and other neurons) can take advantage of the multiple simultaneous codes and process them one by one, or simultaneously in order to perform different tasks. The sender and the content of the signals can also be used separately to discriminate the information received by a neuron by distinctly processing the input as a function of these codes. These mechanisms can contribute to build dynamical invariants through a self-organizing strategy that includes non supervised learning as a function of local discrimination. Artificial CPG networks built with neurons that display neural signatures allow for a new set of learning rules that include not only the modification of the connections, but also the parameters that affect the local discrimination. 


\subsection{Homeostasis: Self-regulation Mechanisms}

CPG research has also shown the presence of many homeostatic mechanisms to self-regulate and to deal with unexpected circumstances at the cellular and neural network levels and in multiple time scales (Marder and Goaillard, 2006). Dynamical invariants that work in short time scales can be built with the above mentioned mechanisms that involve neuron and synapse dynamics and specific topologies. However other types of invariants working in longer time scales can use mechanisms of adaptation and learning (including sub cellular plasticity) to generate rhythms even in the absence of the input that sustains this rhythm under normal circumstances (Thoby-Brisson and Simmers, 1998). Long scale dynamical invariants arise from self-regulatory mechanism that involve tightly regulated synaptic and intrinsic properties. Models could implement this self regulation through specific synaptic and sub cellular learning paradigms.

Implementing already known sub cellular and network learning mechanisms (Marder and Prinz, 2002), CPGs may easily accommodate the degradation process of a working robot. If a joint loses torque, if pieces begin to wear off, a CPG implementing these mechanisms could adjust its function to reflect the natural evolution of the mechanical parts. Even if some part suddenly stops working or is disconnected from the main body of the robot, the CPG could still keep its original function.

\section{CONCLUSIONS}

Central Pattern Generator neural circuits are well known for their ability to generate and sustain rhythmic activity. And they do so in a robust manner, tolerant to perturbations, but with flexibility, being able to adjust their working regime to the requirements of the environment.

We have presented recent results that extend the idea of a CPG. Results from which the robotics field will surely benefit. To begin with, we have introduced a new design idea, termed dynamical invariant. The key aspect of designing an artificial CPG should be specifying what restrictions must be kept under any condition. So, the presence of one or several dynamical invariants will guarantee the effectiveness of motor control in unknown or changing environments. Living CPGs use different strategies to implement their invariants, we have reviewed some of them in this paper.
First, we have reported on studies that conclude that those topologies that maximize rhythm quality are non-open topologies. That is, every neuron in the circuit receives at least one input from the rest of the circuit. Following this, we review those mechanisms that are responsible for rhythm start, stop and sustenance. Few works known to the authors have concerned themselves with the issue of initiation and termination of CPGs in robotics. We believe that the works mentioned here will be of great inspiration to robot designers. Then, we discuss the mechanism by which living motor neurons are believed to code motor commands. Studying it, robotic CPGs can gain greater flexibility and robustness by decoupling synchronization and coding mechanisms. Next, a recently discovered property of bursting neurons is presented: neural signatures. This mechanism increases the computing capabilities of a CPG. It allows neurons to code context specific information so that receivers of different neural messages may discriminate their input according to the identity of the sender, circumstances on which a message occurs, etc. We believe that this result will widen the view of CPGs from simple pattern generators to information processing capable circuits. And finally, we present reports on the ability of long term adaptation of CPGs. Several results indicate that CPGs can satisfy their dynamical invariants despite differences from animal to animal, or development of the animal from young to adult or changes in function and/or size due to illnesses and injuries. Understanding the mechanisms underlying this ability will allow designers to construct CPGs that will adapt over a range of "robot families" and to the consequences of long time operation like loss of performance and degradation of the parts.

To conclude, we believe that biology has still many secrets to reveal. We have pointed out key issues that robotics has not yet explored, but with promising potential. We are beginning to implement some of these ideas in our own robots (HerreroCarrón et al., 2010), and look forward to the next generation of CPGs.

\section{ACKNOWLEDGEMENTS}

Work supported by MICINN BFU2009-08473, CAM S-SEM-0255-2006 and TIN 2007-65989. Fernando Herrero-Carrón with an FPU-UAM grant. 


\section{REFERENCES}

Aguirre, C., Campos, D., Pascual, P., and Serrano, E. (2005). A model of spiking-bursting neuronal behavior using a piecewise linear two-dimensional map. Computational Intelligence and Bioinspired Systems, pages 130-135.

Arshavsky, Y. I., Deliagina, T. G., Orlovsky, G. N., Panchin, Y. V., Popova, L. B., and Sadreyev, R. I. (1998). Analysis of the central pattern generator for swimming in the mollusk clione. Annals of the New York Academy of Sciences, 860(1):51-69.

Brezina, V., Orekhova, I. V., and Weiss, K. R. (2000). The neuromuscular transform: The dynamic, nonlinear link between motor neuron firing patterns and muscle contraction in rhythmic behaviors. J Neurophysiol, 83(1):207-231.

Bucher, D., Prinz, A. A., and Marder, E. (2005). Animalto-animal variability in motor pattern production in adults and during growth. J. Neurosci., 25(7):16111619.

Elson, R. C., Selverston, A. I., Abarbanel, H. D., and Rabinovich, M. I. (2002). Inhibitory synchronization of bursting in biological neurons: Dependence on synaptic time constant. J Neurophysiol, 88(3):1166-1176.

Grillner, S. (2006). Biological pattern generation: The cellular and computational logic of networks in motion. Neuron, 52(5).

Grillner, S., Hellgren, J., Ménard, A., Saitoh, K., and Wikström, M. A. (2005). Mechanisms for selection of basic motor programs-roles for the striatum and pallidum. Trends Neurosci, 28(7):364-370.

Herrero-Carrón, F., Rodríguez, F. d. B., and Varona, P. (2010). Bio-inspired design strategies for cpg control in modular robotics. Submitted.

Hindmarsh, J. L. and Rose, R. M. (1984). A model of neuronal bursting using three coupled first order differential equations. Proceedings Of The Royal Society Of London. Series B, Containing Papers Of a Biological Character. Royal Society (Great Britain), 221(1222):87-102.

Hodgkin, A. L. and Huxley, A. F. (1952). A quantitative description of membrane current and its application to conduction and excitation in nerve. The Journal of physiology, 117(4):500-544.

Huerta, R., Varona, P., Rabinovich, M. I., and Abarbanel, H. D. (2001). Topology selection by chaotic neurons of a pyloric central pattern generator. Biological $C y$ bernetics, 84(1):L1-L8.

Ijspeert, A. (2008). Central pattern generators for locomotion control in animals and robots: A review. Neural Networks, 21(4):642-653.

Kopell, N. and Ermentrout, G. (2000). Mechanisms of phase-locking and frequency control in pairs of coupled neural oscillators.

Kopell, N. and Somers, D. (1995). Anti-phase solutions in relaxation oscillators coupled through excitatory interactions. Journal of Mathematical Biology, 33(3):261280.
Latorre, R., Rodríguez, F., and Varona, P. (2006). Neural signatures: Multiple coding in spiking-bursting cells. Biological Cybernetics, 95(2):169-183.

Marder, E. and Goaillard, J.-M. (2006). Variability, compensation and homeostasis in neuron and network function. Nature Reviews Neuroscience, 7(7):563574.

Marder, E. and Prinz, A. A. (2002). Modeling stability in neuron and network function: the role of activity in homeostasis. BioEssays : news and reviews in molecular, cellular and developmental biology., 24(12):1145-1154.

Oprisan, S. A., Prinz, A. A., and Canavier, C. C. (2004). Phase resetting and phase locking in hybrid circuits of one model and one biological neuron. Biophys $J$, 87(4):2283-2298.

Prinz, A. A., Bucher, D., and Marder, E. (2004). Similar network activity from disparate circuit parameters. Nature Neuroscience, 7(12):1345-1352.

Reyes, M., Huerta, R., Rabinovich, M., and Selverston, A. (2008). Artificial synaptic modification reveals a dynamical invariant in the pyloric cpg. European Journal of Applied Physiology, 102(6):667-675.

Rowat, P. F. and Selverston, A. I. (1997). Oscillatory mechanisms in pairs of neurons connected with fast inhibitory synapses. Journal of Computational Neuroscience, 4(2):103-127.

Rulkov, N. F. (2002). Modeling of spiking-bursting neural behavior using two-dimensional map. Physical Review E, 65(4):041922+.

Selverston, A. B., Szücs, A., Huerta, R., Pinto, R. D., and Reyes, M. (2009). Neural mechanisms underlying the generation of the lobster gastric mill motor pattern. Frontiers in Neural Circuits.

Stiesberg, G. R., Reyes, M. B., Varona, P., Pinto, R. D., and Huerta, R. (2007). Connection topology selection in central pattern generators by maximizing the gain of information. Neural Computation, 19(4):974-993.

Szucs, A., Pinto, R. D., Rabinovich, M. I., Abarbanel, H. D., and Selverston, A. I. (2003). Synaptic modulation of the interspike interval signatures of bursting pyloric neurons. Journal of neurophysiology, 89(3):1363-1377.

Thoby-Brisson, M. and Simmers, J. (1998). Neuromodulatory inputs maintain expression of a lobster motor pattern-generating network in a modulationdependent state: Evidence from long-term decentralization in vitro. J. Neurosci., 18(6):2212-2225.

Wang, X.-J. and Rinzel, J. (1992). Alternating and synchronous rhythms in reciprocally inhibitory model neurons. Neural Comput., 4(1):84-97. 